\title{
Doctor, can I drive? A quality improvement project to improve driving advice given to patients in our cardiology unit
}

\author{
Authors: Simon Pearse ${ }^{A}$ and Patrick Savage ${ }^{B}$
}

\section{Background}

Many cardiac conditions confer a restriction on driving as laid out by the Driver and Vehicle Licensing Agency (DVLA). ${ }^{1}$ As doctors, it is our duty to ensure that patients are correctly informed of these restrictions. ${ }^{2}$ We noted a paucity of information documented and given to patients on discharge in addition to a lack of awareness among staff with respect to the latest DVLA guidance. Using a Plan, Do, Study, Act (PDSA) model, we designed a quality improvement project to address this issue in the cardiology department at Kingston Hospital.

\section{Methods}

Baseline data were collected by first auditing a selection of patients discharged over a period of 2 weeks with either acute coronary syndrome (ACS), syncope or requiring pacemaker insertion. Using a questionnaire, we then evaluated our doctors' knowledge of current DVLA guidance. We then re-audited this data across two cycles of change with the following interventions:

Cycle 1: Introduction of DVLA guidance posters to the ward with information specific to our patient group. Dissemination of quick links to full guidance online and staff education with respect to this guidance.

Cycle 2: Further consolidation of cycle 1 interventions and introduction of patient information leaflets. Introduction of DVLA guidance lanyards with clear, succinct information relevant to our patient group.

Results

Prior to implementing change, we found that driving advice given to patients was poorly documented in both discharge letters and patient notes $(0 \%, n=15)$. This improved to $40 \%(n=15)$ after one cycle then to $67 \%(n=12)$ after the second cycle. Questionnaire scores improved from $20 \%(n=6)$ at baseline to $80 \%(n=5)$ after cycle two.

Authors: ${ }^{A}$ Kingston NHS Trust; ${ }^{B}$ Kingston Hospital NHS Foundation Trust

\section{Conclusion}

Cardiac conditions often restrict patients' ability to drive and we often don't advise our patients appropriately. We have demonstrated a sustained positive change using simple interventions delivered across two cycles of change which has led to better knowledge of DVLA guidance among our doctors and a dramatic increase in appropriate advice given to our patients on discharge.

\section{References}

1 Driver and Vehicle Licensing Agency. Assessing fitness to drive: guide for medical professionals. DVLA, February 2019. Available from: www.gov.uk/government/publications/assessing-fitness-to-drive-aguide-for-medical-professionals [Accessed 18 Feb 2019].

2 General Medical Council. Good medical practice. London: GMC, 2014. Available from: www.gmc-uk.org/ethical-guidance/ethicalguidance-for-doctors/good-medical-practice [Accessed 18 Feb 2019]. 